\title{
An STM for Molecules and Wide-Bandgap Crystal ${ }^{1}$
}

\author{
H. Akagia ${ }^{a}$, L. Arissian ${ }^{a, c}$, J. B. Bertrand ${ }^{a}$, P. B. Corkum ${ }^{a}, *$, M. Gertsvolf ${ }^{a}$, D. Pavicic ${ }^{a}$, \\ D. M. Rayner ${ }^{a}$, C. Smeenk ${ }^{a}$, A. Staudte ${ }^{a}$, D. M. Villeneuve ${ }^{a}$, and H. J. Wörner ${ }^{a}$ \\ ${ }^{a}$ Joint Laboratory for Attosecond Science, National Research Council of Canada and University of Ottawa, \\ 100 Sussex Drive, Ottawa, ON Canada K1A OR6 \\ *e-mail:Paul.Corkum@nrc_cnrc.gc.ca \\ ${ }^{b}$ Quantum Beam Science Directorate, Japan Atomic Energy Agency, Tokai-mura, Naka-gun, Ibaraki 319-1195, Japan \\ ${ }^{c}$ Department of Physics, Texas A\&M University, 4242 TAMU, College Station, TX 77843-4242 USA \\ Received March 20, 2009
}

\begin{abstract}
We are all influenced by Nicolai Delone's research. Through much of his career, his influence reached Canada through his published papers. However, Professor Delone visited the National Research Council of Canada's laboratories at least three times from about 1989 to 1995 . At that time he was primarily interested in stabilization. Stabilization refers to the fact that, as the intensity of laser light illuminating an atom is increased, the ionization rate passes through a maximum, then falls. Surprisingly the atom becomes stable against ionization at very high intensities.
\end{abstract}

PACS numbers: $33.20 . \mathrm{Xx}, 82.50 . \mathrm{Hp}$

DOI: $10.1134 / \mathrm{S} 1054660 \mathrm{X} 09150018$

In addition to enthusiastically arguing the merits of stabilization, on one of his trips Professor Delone brought slides of a canoe trip he took with his family to see the famous wooden churches of Northern Russia. He showed the slides to a group of us at my (Corkum) house. On that occasion, he presented me with one of his paintings showing a boat beached near small sheds in the wilderness. The painting still hangs in my home as a pleasant reminder of him. I show it to all of my scientific visitors. It is included as Fig. 1.

In our lab, Professor Delone is best known for his contributions to atomic tunnelling [1] one of the most fundamental of all quantum mechanical processes. As a result of the development of the scanning tunnelling microscope (STM) tunnelling has transformed surface science [2]. To Professor Delone and his colleagues, electrons can also tunnel from atoms or molecules when illuminated with intense infrared radiation. Professor Delone's prediction for the structure of the continuum electron wave packet created by laser tunnelling from atoms [3] is

$$
\Psi\left(p_{\perp}\right)=\left\langle p_{\perp} \mid \Psi_{1}\right\rangle \exp \left(-\frac{\sqrt{2 I_{p}}}{E} p_{\perp}^{2}\right)
$$

where $\Psi\left(p_{\perp}\right)$ is the perpendicular component of the electron's momentum wavefunction in the continuum after tunnelling, $\Psi_{1}$ is the wavefunction of the bound electron, $I_{p}$ is the ionization potential of the atom or molecule and $E$ is the electric field (all quantities in atomic units).

\footnotetext{
${ }^{1}$ The article is published in the original.
}

In memory of the life of Professor Delone and his many contributions to science, we describe our vision and our recent work on tunnelling. Equation (1) shows that the electron that tunnels from the atom or molecule contains a filtered image of the bound electron wavefunction. In the molecular case, if we could rotate the molecule we could sample all possible projections of the orbital, gaining all information on the orbital [4]. Returning to the STM, rotating a molecule and measuring the ionization probability is almost a direct analogue to scanning a tip across a solid and measuring the tunnelling current.

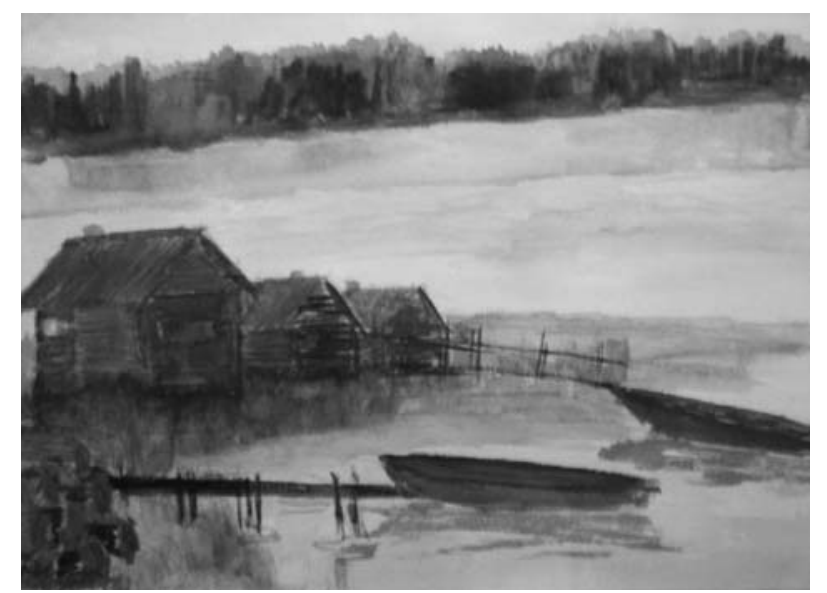

Fig. 1. Painting by Professor Delone. 
(a)

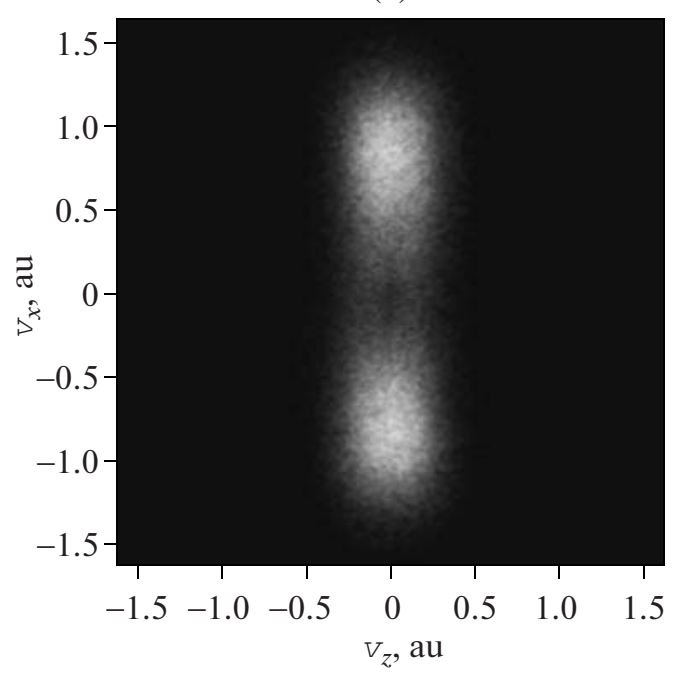

(b)

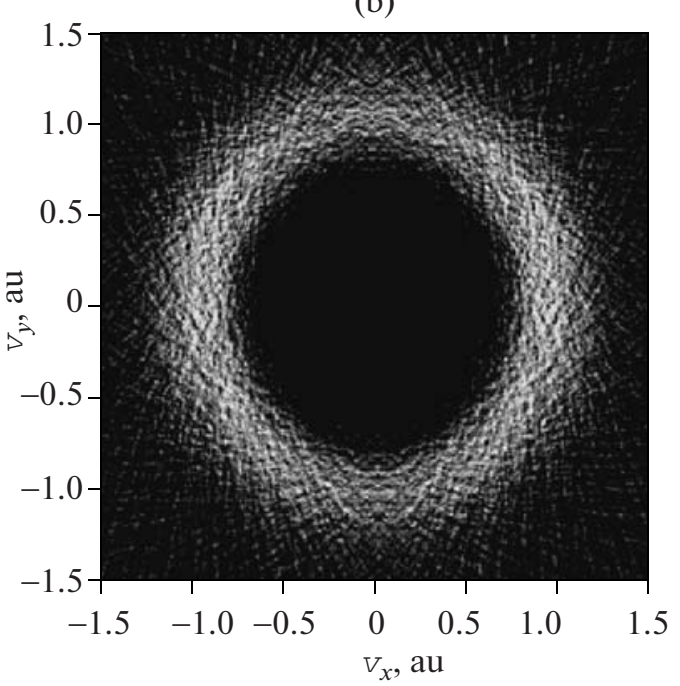

Fig. 2. Electron velocity distribution in elliptically polarized light. The laser propagates along the $V_{z}$ axis and the polarization rotates in the $v_{x}-v_{y}$ plane. The two projections are taken in Argon with $50-\mathrm{fs} 800 \mathrm{~nm}$ laser pulses of intensity $2.5 \times 10^{14} \mathrm{~W} / \mathrm{cm}^{2}$.

However, our molecular STM contains much more information than just the tunnelling probability. Since the electron escapes into vacuum, we can measure $\left|\Psi\left(p_{\perp}\right)\right|^{2}$ directly.

The paper is divided into four sections. In the first, using atomic ionization as the example, we describe how we can make measurements without re-collision. In the second section we show one approach to measuring the tunnelling probability as a function of molecular alignment using a correlation method [5]. The third section concentrates on how small molecules can be aligned with short laser pulses. Once molecules can be aligned, we can also measure the tunnelling rate as a function of alignment by measuring the ionization probability with linearly polarized light. We use $\mathrm{Br}_{2}$ and $\mathrm{N}_{2}$ as examples in Section 3.

Finally we complete the circle, returning to solids. A traditional STM is confined to the surface of solids. Laser radiation does not suffer from this limitation in large band gap materials. One might expect that the ionization probability would change as a function of the angle between the light polarization and the crystal axis much as it does for a molecule. We complete our review by demonstrating that tunnelling can be used to identify the symmetry of a crystal - with micron spatial precision-anywhere in the bulk [6].

\section{MEASURING THE NASCENT ELECTRON DISTRIBUTION USING CIRCULARLY POLARIZED LIGHT}

When an intense infrared laser pulse irradiates an atom or molecule, the field significantly distorts the Coulomb potential allowing the valence electron(s) to tunnel ionize. The free electron's wavefunction immediately after tunnel ionization is then given by Eq. (1).
If we can recover the nascent shape of the tunnelled electron wave packet for different angles of the laser polarization, then Eq. (1) shows that we fully determine the bound wavefunction. Once the electron is free, its motion is determined by the laser field or any encounters it might have with charged particles.

In linearly polarized light the free electron may reencounter its parent nucleus on one or more occasions due to the oscillating field. This interaction between the electron and the positively charged ion distorts the wavefunction from its nascent shape. In circularly polarized light this behaviour (often termed Coulomb focusing [7]) is avoided. If the laser field (in atomic units) is $E=E_{0}[\cos \omega t \hat{i}+\sin \omega t \hat{j}]$ then the electron's velocity is

$$
\begin{aligned}
v(t) & =E_{0} / \omega\left[\left(\sin \omega t-\sin \omega t_{i}\right) \hat{i}\right. \\
& \left.+\left(\cos \omega t_{i}-\cos \omega t\right) \hat{j}\right],
\end{aligned}
$$

where $t_{i}$ is the time of ionization. Equation (2) shows that the free electron moves along a cycloid-like trajectory with a constant drift velocity directed away from the nucleus and perpendicular to the direction of the field at the instant of ionization. An observation after the laser pulse has passed will measure the drift velocity due to the phase of the field at $t_{i}$ [8].

In circularly (or elliptically) polarized light, the action of the laser field leaves the momentum distribution undisturbed only along $\mathbf{k}$ - the direction of light propagation. Viewing the electron distribution along the $\mathbf{k}$-vector axis is therefore a natural test of Eq. (1). Knowing the bound state wavefunction (for example of a rare gas atom) we can test the filter function (i.e., the exponential term in Eq. (1)). Knowing the filter 


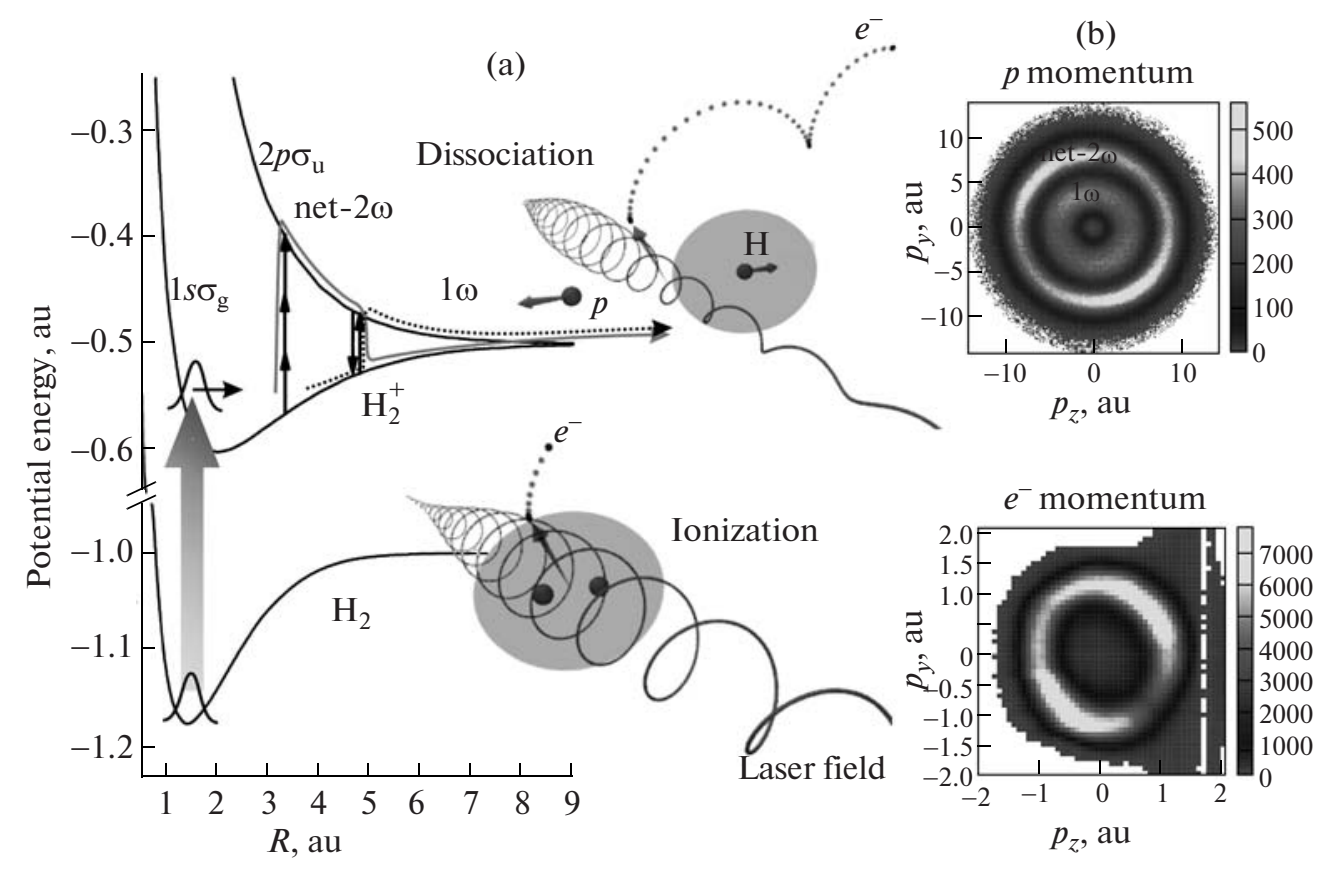

Fig. 3. Illustration of alignment free determination of molecular frame tunnelling probability in an intense laser pulse for the example of $\mathrm{H}_{2}$. (a) First, the electron tunnel ionizes, and is driven away from the molecule in a direction determined by the laser field. Second, the molecular bond of the parent ion is bond softened by the same laser field leading to dissociation of the fragments. This fast dissociation freezes the orientation of the molecular axis in the laboratory frame. (b) $\mathrm{H}^{+}$momentum (top) spectrum illustrates the experimental signature of bond softening. The lower panel shows the corresponding electron momentum. From this information the molecular frame ionization probability can be reconstructed.

function, we can gain insight into the wavefunction from which an electron tunnels.

We have begun an experiment using elliptically (almost circular) polarized light to ionize argon. In the experiment the laser propagates along the $Z$ axis and polarization rotates in the $X-Y$ plane. The laser intensity was $2.5 \times 10^{14} \mathrm{~W} / \mathrm{cm}^{2}$. Figure $2 \mathrm{a}$ shows the momentum distribution in the $v_{z}-v_{x}$ plane as measured by a velocity map imaging experiment [9]. In Fig. 2a the distribution along the k-vector axis (i.e., along $v_{z}$ ) is well described by a Gaussian function whose standard deviation is given by Eq. (1). Delone's idea of tunnel ionization acting as a filter on the bound wavefunction provides a clear explanation for this observation.

Figure $2 \mathrm{~b}$ shows a similar distribution measured in the $v_{x}-v_{y}$ plane: the plane of polarization. A small ellipticity of the laser field appears as a slightly larger ionization yield along the $v_{x}$ axis compared to $v_{y}$. A close examination also reveals the velocity distribution is elliptical with a major axis along $v_{y}$ and minor axis along $v_{x}$. If our target gas was switched to an aligned molecule then we would see an additional modulation caused by the angle dependent tunnelling probability. In the next section we will show how this experiment can be generalized to measure angle dependent tunnelling very accurately.

\section{MEASURING THE ANGLE DEPENDENT TUNNEL IONIZATION PROBABILITY OF $\mathrm{H}_{2}$}

Angle-dependent ionization has been studied using molecules that have been aligned in the laboratory frame with a linearly polarized laser pulse [10]. Once aligned, the ionization probability as a function of the angle between a second linearly polarized pulse and the molecular axis is readily obtained $[11,12]$. This is an important approach that we elaborate in the following section. However, this technique has limitations. In general, the angular resolution is limited, especially for weakly anisotropic molecules such as $\mathrm{H}_{2}$ [13]. Furthermore this approach has so far proven to be insensitive to the orientation of heteronuclear molecules. Along the lines of the preceding paragraph we have developed a different experimental approach to measure the angular tunnel ionization rate in the molecular frame. It is independent of the experimentally achievable degree of alignment and orientation. Here, we compare the experimental results of the angle dependent ionization rate in $\mathrm{H}_{2}$ and $\mathrm{D}_{2}$.

Figure 3 illustrates our concept of an alignment free single molecule STM on the example of $\mathrm{H}_{2}$. Instead of actively aligning the hydrogen molecules, i.e., fix the molecules in space before tunnelling, we ionize a randomly aligned ensemble of molecules and determine the molecular axis after tunnelling. 

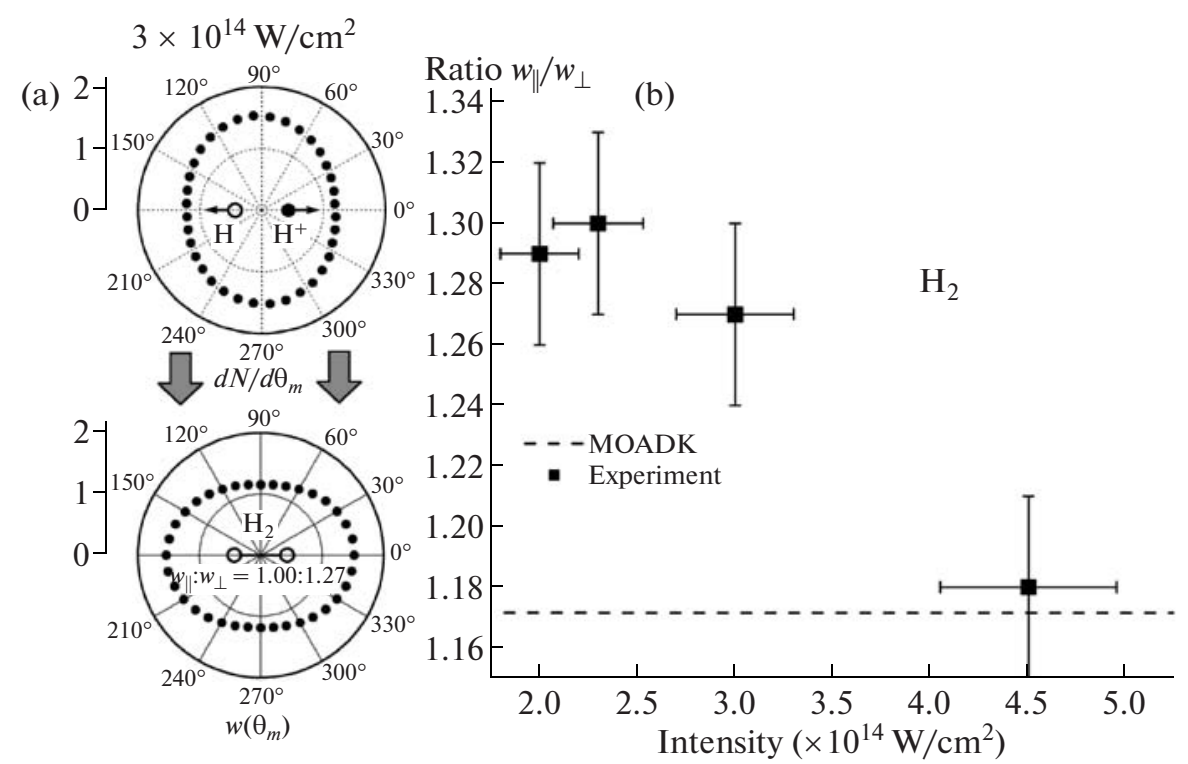

Fig. 4. Single molecule STM of $\mathrm{H}_{2}$. (a) Top panel: Photoelectron angular distribution in the molecular frame. Lower panel: Normalized molecular frame tunnelling probability. (b) Intensity dependence of the parameterized tunnelling anisotropy in $\mathrm{H}_{2}$ compared to the molecular ADK model [16].

A circularly polarized femtosecond laser pulse first ionizes a randomly aligned $\mathrm{H}_{2}$ molecule (Fig. 3a). As described in Eq. (2), the electron is being accelerated in the laser field and drifts away on a cycloid trajectory. Second, the newly created molecular ion is bond softened by the laser pulse resulting in the dissociation of $\mathrm{H}_{2}^{+}$into a proton and a hydrogen atom. Bond softening [14] is a general mechanism by which molecules in strong laser fields dissociate. In the case of $\mathrm{H}_{2}^{+}$the laser field strongly couples the two lowest lying electronic surfaces at certain internuclear separations. The two different pathways to dissociation result in protons that are well separated in momentum space. An example of that momentum space projected onto the plane of polarization is shown in the top panel of Fig. $4 \mathrm{~b}$.

Whereas the direction of the observable electron momentum labels the field direction at the moment of ionization, the direction of the proton labels the orientation of the molecule at the instant of the ionization. By measuring electrons and ions in coincidence, we obtain the angle between the field direction at the moment of ionization and the molecular axis for each event, i.e., each single molecule.

To achieve this experimentally, we employed Cold Target Recoil Ion Momentum Spectroscopy (COLTRIMS) [15]. COLTRIMS allows the simultaneous measurement of the 3 dimensional momentum components of electrons and ions in coincidence. To induce tunnelling in $\mathrm{H}_{2}$ molecules we focused circularly polarized Ti:Sa laser pulses ( $40 \mathrm{fs}, 800 \mathrm{~nm}$, up to $5 \mu \mathrm{J}, 60 \mathrm{kHz}$ repetition rate) to peak intensities of $(1.0-4.5) \times 10^{14} \mathrm{~W} / \mathrm{cm}^{2}$ into a gas jet. The gas jet was produced in a supersonic expansion of $\mathrm{H}_{2}$ gas at $40 \mathrm{~K}$ which results in an internal translational temperature in the gas jet of $\ll 10 \mathrm{~K}$.

The top panel of Fig. 4a shows the photoelectron angular distribution in the frame of the detected proton. To obtain the molecular frame tunnelling probability $w\left(\theta_{m}\right)$ of the neutral molecule (shown in the lower panel of Fig. 4a) this measured distribution must be rotated by approx. $90^{\circ}$ according to the classical equations of motion of an electron in a rotating electric field. The lower panel in Fig. 4a can be regarded as the tunnelling current when the "tip" of the microscope, i.e., the laser field, rotates about the molecule. The sense of rotation depends on the helicity of the laser polarization. However, for $\mathrm{H}_{2}$ this is not important. Only when applying this approach to heteronuclear molecules the laser helicity needs to be taken into account.

Finally, in Fig. 4b we present the measured intensity dependence of the ratio of tunnelling probabilities along and perpendicular to the molecular axis and compare our results with the prediction of molecular ADK (MOADK) theory [16]. The figure shows how this approach can be used to test existing tunnelling theory. For example, MOADK does not nearly reproduce the observed intensity dependence. Parenthetically we want to note that, despite the shown large underestimation of tunnelling anisotropy by an established theory, the experiment only provides a lower bound for this anisotropy [5].

While it should be easy to apply this technique to any small heteronuclear molecule that bond softens, not all molecular ions will bond soften from the ground state. One universal process for measuring molecular alignment or orientation is Coulomb 
Explosion Imaging. It operates for all small molecules [17]. Thus Coulomb explosion solves the problem of determining molecular orientation or alignment, but at the expense of a new problem - we must identify the electron of interest from the electrons created by the explosion. This can be achieved by using long wavelength light for the initial ionization and short wavelength for coulomb explosion. The electron of interest is then uniquely labeled by its large momentum $p_{0}=E_{0} / \omega$ provided the frequencies are sufficiently different to overcome the different intensity required for single ionization and Coulomb explosion.

\section{MOLECULAR ALIGNMENT}

Field-free laser alignment of molecules in the laboratory [10] allows one to observe physical processes in the molecular frame. This ability, for instance, has led to the imaging of molecular orbitals using high harmonic generation (HHG) [18] and the observation of laser-induced electron diffraction [4]. A better understanding of these strong-field phenomena requires a direct measurement of their first common step: tunnel ionization. To achieve this, we use non-adiabatic alignment and measure the tunnel ionization yield as the molecule is rotated with respect to the direction of the (linear) polarization of the ionizing laser pulse.

Under the influence of a strong, non-resonant and non-ionizing laser pulse, molecules tend to align their most polarizable axis along the polarization of the field. An anisotropic well of depth $H=-\Delta \alpha E_{0}^{2} / 4$ is thus created, where $E_{0}$ is the maximum electric field strength and $\Delta \alpha$ is the difference between the parallel and perpendicular polarizability components of the molecule [10]. Typically, $H \approx 1.25 \mathrm{eV}$ for $I_{0}=5 \times$ $10^{13} \mathrm{~W} / \mathrm{cm}^{2}$ and $\Delta \alpha=10 \AA^{3}$.

If the pulse duration is considerably shorter than the rotational period of the molecule, a nonadiabatic interaction takes place: rotational states of the molecule are being coherently populated by a Raman excitation from the aligning pulse, thus creating a rotational wave-packet. The degree of alignment obtained will depend on the strength of the interaction, as given by $H$, but also on the rotational temperature of the gas.

The quantity commonly used to evaluate the degree of alignment is the expectation value of $\cos ^{2} \theta$ weighted by the alignment distribution over the molecule's ensemble, where $\theta$ is the angle between the aligned molecular axis and the laser polarization. For an isotropic distribution, $\left\langle\cos ^{2} \theta\right\rangle=1 / 3$. In nonadiabatic alignment, $\left\langle\cos ^{2} \theta\right\rangle$ will first peak after the interaction with the aligning pulse, this is the prompt alignment. It will subsequently modulate strongly at fractional revival times [19], therefore offering field-free aligned molecules.

The level of agreement between ionization rate measured in the laboratory and the one in the molec- ular frame depends on the degree of alignment achieved. Only perfect alignment allows exact correspondence between the two frames. Otherwise, the molecular frame signal is convolved with the actual alignment distribution. However, if the degree of alignment is known, the molecular profile can be retrieved by applying the appropriate deconvolution on the lab measurement $[11,12]$. We will describe and apply this technique to obtain the ionization probability as a function of angle in $\mathrm{N}_{2}$ and $\mathrm{Br}_{2}$.

We have developed an experimental configuration that is compatible with the high gas densities $\left(\sim 10^{17} / \mathrm{cm}^{3}\right)$ that are required to observe HHG. This allows us to perform the measurement of the ion yield in parallel to the measurement of the high harmonic yield. We perform our measurements in a HHG source chamber [20]. A pulsed gas jet $(50 \mathrm{~Hz})$ is used with a stagnation back pressure of $1 \mathrm{~atm}$. This provides rotational cooling through a supersonic expansion to about $30 \mathrm{~K} 1 \mathrm{~mm}$ below the nozzle, where we focus $(f=50 \mathrm{~cm})$ the $800 \mathrm{~nm}(50 \mathrm{~Hz})$ pump and probe laser pulses.

First, we nonadiabatically align the molecules. Then, we ionize them with a second intense $\sim 1.5 \times$ $10^{14} \mathrm{~W} / \mathrm{cm}^{2} 30$-fs linearly polarized pulse. We do this experiment with $\mathrm{N}_{2}$ then $\mathrm{Br}_{2}$ molecules. The ionization is measured by observing voltage signal through a capacitor from a dc-biased $(-200 \mathrm{~V})$ mesh collecting the ions downstream $(\sim 10 \mathrm{~cm})$ in the gas expansion. We measure the total ion yield that is, under our conditions, dominated by the singly charged molecular ions. The signal, (see experimental data in Fig. 5), is averaged over 500 laser shots. We change the alignment of the molecules by rotating the polarization of the aligning beam with a motorized half-wave plate. The delay $\Delta t$ separating both laser pulses is chosen in order to have maximal alignment under field-free conditions: $\Delta t=4.12$ ps for $\mathrm{N}_{2}$ (maximal alignment corresponding to the first rotational half-revival) and $\Delta t=$ $0.5 \mathrm{ps}$ (prompt alignment) for $\mathrm{Br}_{2}$.

$\mathrm{N}_{2}$ is aligned with a $50-\mathrm{fs} 800 \mathrm{~nm}$ linearly polarized pulse of peak intensity $5 \times 10^{13} \mathrm{~W} / \mathrm{cm}^{2}$. For better alignment in $\mathrm{Br}_{2}$, we stretch the aligning pulse to $100 \mathrm{fs}$ and estimate its intensity to $1.5 \times 10^{13} \mathrm{~W} / \mathrm{cm}^{2}$. This intensity is maximal in both $\mathrm{N}_{2}$ and $\mathrm{Br}_{2}$ experiments so that no ions are observed. In $\mathrm{N}_{2}$, wave-packet calculations predict a maximum value of $\left\langle\cos ^{2} \theta\right\rangle=0.52$ in our experimental conditions [20]. For $\mathrm{Br}_{2}$ we estimate $\left\langle\cos ^{2} \theta\right\rangle=0.5$ [21]; although the $\mathrm{Br}_{2}$ polarizability is greater than for $\mathrm{N}_{2}\left(\Delta \alpha_{\mathrm{Br}_{2}} / \Delta \alpha_{\mathrm{N}_{2}} \sim 3.5\right.$ [22] $)$, the alignment pulse intensity is lowered to avoid ionization.

The angular ionization yield $M(\beta)$, where $\beta$ is the angle between the polarization axes of the aligning and the ionizing beams, is the convolution of the molecu- 

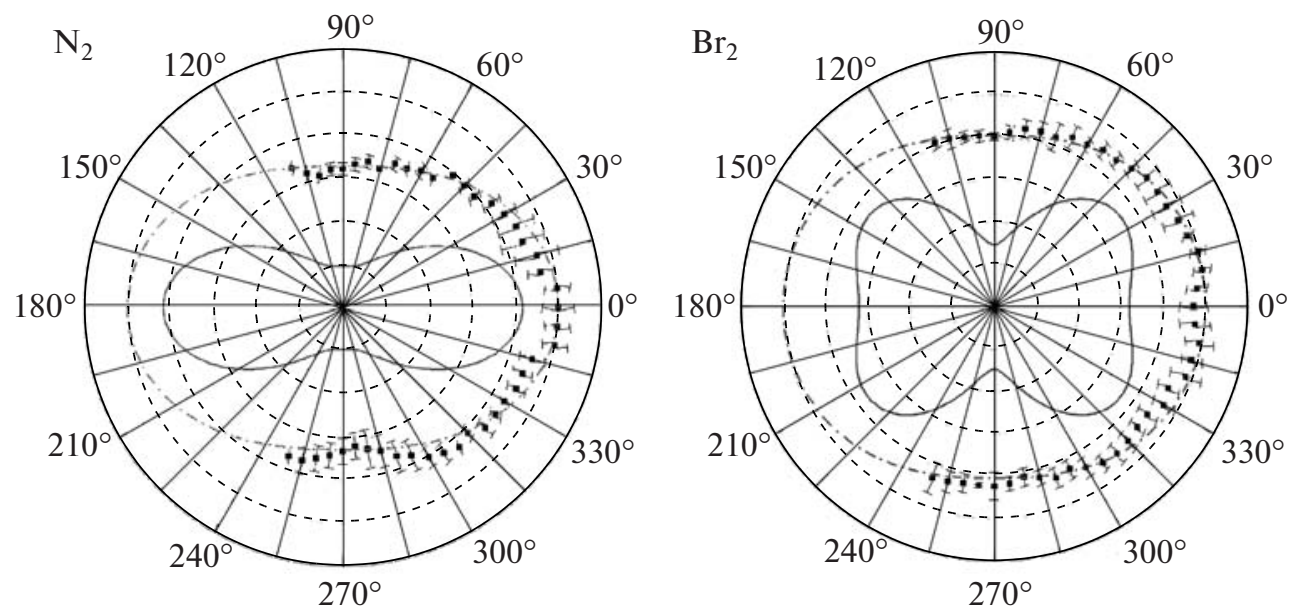

Fig. 5. The measured ionization yield $M(\beta)$ (scatter) as a function of the angle $\beta$ between the polarization axes of the aligning and the ionizing beams are displayed for both $\mathrm{N}_{2}$ (left) and $\mathrm{Br}_{2}$ (right). The calculated best fit $M(\beta)$ (dash-dotted) and the corresponding molecular frame angular ionization probability $S(\beta)$ (solid, arb. normalized) are also plotted. $\mathrm{N}_{2}\left(\mathrm{Br}_{2}\right)$ was aligned with a linearly polarized pulse of $50 \mathrm{fs}(100 \mathrm{fs})$ duration and peak intensity $5 \times 10^{13} \mathrm{~W} / \mathrm{cm}^{2}\left(1.5 \times 10^{13} \mathrm{~W} / \mathrm{cm}^{2}\right)$ and ionized with a $30-\mathrm{fs}$ pulse of peak intensity $1.6 \times 10^{14} \mathrm{~W} / \mathrm{cm}^{2}\left(1.5 \times 10^{14} \mathrm{~W} / \mathrm{cm}^{2}\right)$.

lar frame angular ionization probability $S(\theta)$ with the alignment distribution $A\left(\theta^{\prime}, \varphi^{\prime}\right)$ :

$$
\begin{gathered}
M(\beta)=C \int_{\varphi^{\prime}=0 \theta^{\prime}=0}^{2 \pi} \int^{\pi} S\left(\theta\left(\theta^{\prime}, \varphi^{\prime} ; \beta\right)\right) A\left(\theta^{\prime}, \varphi^{\prime}\right) \\
\quad \times \sin \theta^{\prime} d \theta^{\prime} d \varphi^{\prime},
\end{gathered}
$$

where $\theta^{\prime}$ and $\varphi^{\prime}$ are the polar and azimuthal angles in the frame about the polarization axis of the aligning beam. To perform the integral, Eq. (3), the following relation is used to relate the molecular $(\theta)$ and lab $(\beta)$ frames through the alignment distribution coordinates $\left(\theta^{\prime}, \varphi^{\prime}\right): \cos \theta=\cos \beta \cos \theta^{\prime}-\sin \beta \sin \theta^{\prime} \sin \varphi^{\prime}$. For symmetric top molecules like $\mathrm{N}_{2}$ and $\mathrm{Br}_{2}, A\left(\theta^{\prime}, \varphi^{\prime}\right)$ is cylindrically symmetric around the polarization axis of the aligning beam, i.e., $A\left(\theta^{\prime}, \varphi^{\prime}\right)=A\left(\theta^{\prime}\right) / 2 \pi$. We use a monotonic profile for $A\left(\theta^{\prime}\right)$ with parameters $\{a=-3.1$, $\varepsilon=2.315\}$ as described in [12] such that $\left\langle\cos ^{2} \theta^{\prime}\right\rangle=0.5$. The angle-dependent ionization probability $S(\theta)$, where $\theta$ is the angle between the molecular internuclear axis and the ionizing beam polarization, is obtained by finding the best fit for $M(\beta)$ to the experimental data. We parametrize $S(\theta)$ as a linear combination of Legendre polynomials,

$$
S(\theta)=\sum_{i=0}^{3} a_{2 i} P_{2 i}(\cos \theta)
$$

and find the following values to best fit the experimental data: for $\mathrm{N}_{2},\left\{a_{0}=0.75, a_{2}=0.85, a_{4}=0.3, a_{6}=0\right\}$ and for $\mathrm{Br}_{2},\left\{a_{0}=0.5, a_{2}=0.3, a_{4}=-0.2, a_{6}=0\right\}$. We found that, for $\mathrm{N}_{2}$, the ionization is about $4+/-1$ times more likely along the internuclear axis than perpen- dicular to it, see (solid line) Fig. 5. This is consistent with previous measurements [11, 12]. In $\mathrm{Br}_{2}$, we observe that the ionization peaks instead around $35^{\circ}$, then is slightly suppressed at $0^{\circ}$ and even more suppressed at $90^{\circ}$.

The deconvolved angular ionization profiles for $\mathrm{N}_{2}$ and $\mathrm{Br}_{2}$ differ substantially from each other. The measured $S(\theta)$ for $\mathrm{N}_{2}$ and $\mathrm{Br}_{2}$ reveal their respective $\sigma_{g}$ and $\pi_{g}$ symmetries. The tunnel ionization yield is clearly sensitive to the symmetry of the highest occupied molecular orbital (HOMO), just like an STM is to the electronic density on a surface. The result for $\mathrm{N}_{2}$ agrees with previous experiments [11, 12], but the tunnel ionization yield for $\mathrm{Br}_{2}$ is broader than that observed in $\mathrm{O}_{2}$ and much broader than in $\mathrm{CO}_{2}$ [12], although all three molecules have a HOMO of $\pi_{g}$ symmetry.

Finally, these results can be generalized in a COLTRIMS [15] system to measure 2D electron distribution. A reader is referred to [4] for details. They can also be generalized to crystal solids [6]. We now skip to solids.

\section{MEASURING TUNNELLING EFFECTS IN SOLIDS}

In transparent solids the band gap is comparable to the ionization potential of molecules. Therefore, many aspects in the interaction of the femtosecond laser pulses with transparent dielectrics and molecular gases are similar. The ionization in solids transfers the electron from the valence to the conduction band and is equivalent to tunnelling in molecules. Non-sequential double ionization in gases is like impact ionization 

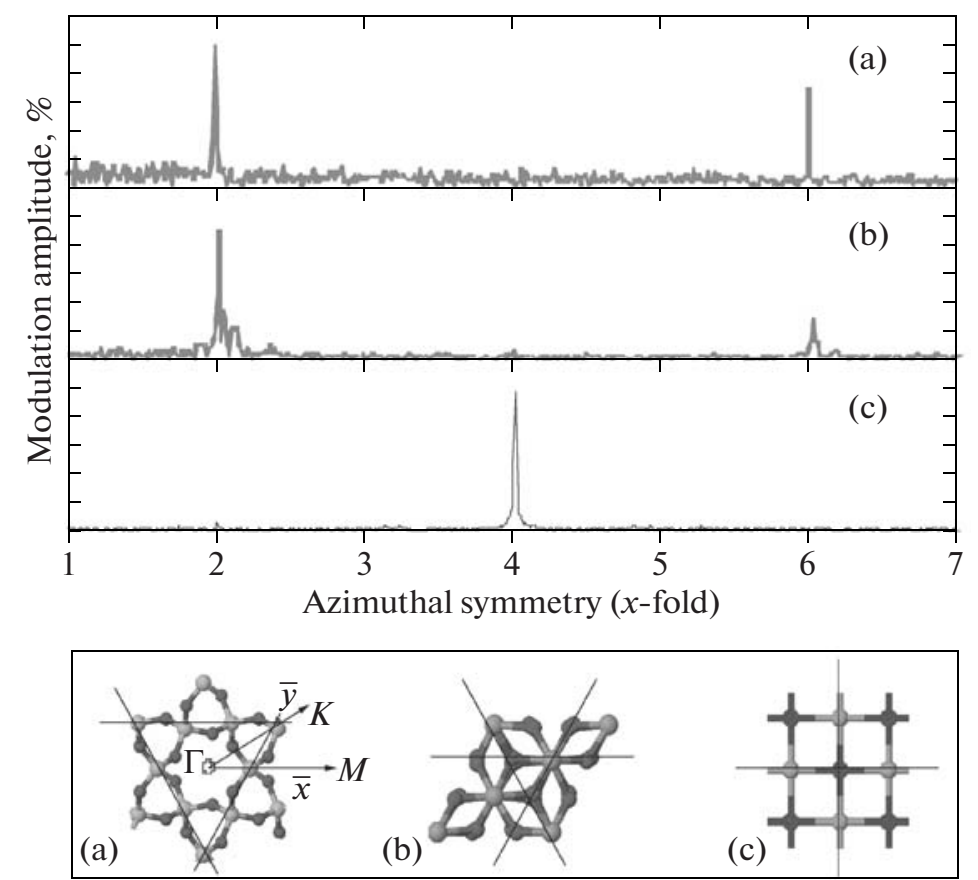

Fig. 6. Nonlinear transmission modulation spectra for (a) quartz, (b) sapphire and (c) lithium fluoride. The vertical scale is $0-$ $0.75 \%$ for $(\mathrm{a}, \mathrm{b})$ and $0-1.5 \%$ for $(\mathrm{c})$. Corresponding crystal structures (the bottom panel) are shown looking down the $z$-axis with dashed lines as eye-guides for the symmetry.

or avalanche in solids, except in solids the collisions are not restricted to the parent ion. The analog to the enhanced ionization in gases is the effect of the Coulomb field from the neighbouring holes that locally changes ionization probability.

However, the metrology tools that are available for the studies of the tunnelling ionization in gases, presented in the previous sections, are not applicable inside solids. The sub-products of the ionization such as high harmonics generation, ions and electrons are confined in the interaction region. The only gauge that is readily available is the transmitted part of the ionizing laser light. The changes in the transmitted intensity, spectrum and polarization carry the information on the ionization process itself. Here the high density of solids facilitates the measurements due to the strong depletion of the laser pulse energy.

We use the self limiting propagation model to describe the ionization process inside solids [23]. The model allows us to get quantitative results for multiphoton and avalanche ionization rates in dielectrics from the measurements of the absorption as a function of the incident laser pulse energy [6].

We have used transmission measurements to study the properties of the ionization in crystalline dielectrics. The crystal lattice is equivalent to the ensemble of aligned molecules and we can measure the changes in the ionization rate as a function of the alignment angle between the laser electric field and the molecular axis. Experimentally we use linearly polarized laser light focussed tightly inside the crystal with microscope objective. The laser input pulse energy is set to give $\sim 20 \%$ absorption. All transmitted light is collected on the photodiode. The sample is moved between laser shots to provide fresh material every time. The laser polarization is continuously rotated relative to the crystal optical axis, $z$. The resulting modulation in the transmitted light is analyzed using Fourier transform. Using this Lock-In like approach we can detect minute modulations in the signal with very high signal to noise ratio. We repeated the experiment for crystals with different lattice symmetries and the results are shown in Fig. 6.

In Fig. 6 the height of the peaks in the Fourier spectrum corresponds to the modulation amplitude. The abscissa is the recurrence frequency of the alignment angle, where 2 corresponds to a rotation period of pi (or two-fold rotational symmetry), 4-pi/2, 6-pi/3. We find that the transmitted signal (and therefore absorption) is modulated with the periodicity that corresponds to the lattice symmetry. Trigonal quartz and sapphire show modulation with 6-fold symmetry and cubic lithium fluoride shows strong 4 -fold peak. The 2-fold peaks in quartz and sapphire spectrum do not probe the crystal structure, but arise due to the residual birefringence in the crystal that is experienced by the peripheral beams in our tight focusing geometry.

The modulation in laser absorption is due to the variation in the ionization probability along different directions in the crystal. From transmission experi- 
ments in aligned quartz we found that the absorption strength is defined by the electron reduced mass tensor in the solid, $m^{*}$. As a result, and in contrast with the molecular case, in solids the ionization probability depends exponentially on the orientation of the laser field with respect to the lattice as shown in Eq. (5):

$$
W(I) \approx \exp \left[-\frac{(2 \Delta)^{3 / 2} \sqrt{m^{*}}}{3 E}\right],
$$

where $\Delta$ is the band gap energy and $E$ is the laser field amplitude (all quantities in atomic units).

This novel application of the properties of tunnelling ionization inside solids promises new technologies. Crystal structure measurements based on a highly nonlinear ionization inside solid provides um-scale spatial resolution. It is, therefore, a microscopy tool. The use of few cycle femtosecond laser pulses gives strong absorption with very little carrier density injection, making it compatible with use on biological materials and possibly live cells. With the femtosecond lasers becoming available in the near infrared range, this technique can be extended to regular semiconductors.

\section{CONCLUSIONS}

Already, atomic and molecular tunneling are critical components of attosecond technology [24] and a source of attosecond dynamics [25]. Above, we have shown that tunnelling offers a new and important diagnostic of the electronic structure of matter. Ours are the first few steps in what will become an important technology and a significant legacy of Professor Delone's research.

\section{REFERENCES}

1. M. V. Ammosov, N. B. Delone, and V. Krainov, Sov. Phys. JETP 64, 1191 (1986).

2. G. Binning, H. Rohrer, C. Gerber, and E. Weibel, Phys. Rev. Lett. 49, 57 (1982).

3. N. B. Delone and V. P. Krainov, J. Opt. Soc. Am. B 8, 1207 (1991).

4. M. Meckel, D. Comtois, D. Zeidler, A. Staudte, D. Pavicic, H. C. Bandulet, H. Pépin, J. C. Kieffer, R. Dörner, D. M. Villeneuve, and P. B. Corkum, Science 320, 1478 (2008).

5. A. Staudte, S. Patchkovskii, D. Pavicic, H. Akagi, O. Smirnova, D. Zeidler, M. Meckel, D. M. Ville- neuve, R. Dörner, M. Yu. Ivanov, and P. B. Corkum, Phys. Rev. Lett. 102, 033004 (2009).

6. M. Gertsvolf, H. Jean-Ruel, P. P. Rajeev, D. D. Klugg, D. M. Rayner, and P. B. Corkum, Phys. Rev. Lett. 101, 243001 (2008).

7. T. Brabec, M. Yu. Ivanov, and P. B. Corkum, Phys. Rev. A 54, R2551 (1996).

8. P. B. Corkum, N. H. Burnett, and F. Brunel, Phys. Rev. Lett. 62, 1259 (1989).

9. A. Eppink and D. Parker, Rev. Sci. Instrum. 68, 3477 (1997).

10. H. Stapelfeldt and T. Seideman, Rev. Mod. Phys. 75, 543 (2003).

11. I. V. Litvinyuk, K. F. Lee, P. W. Dooley, D. M. Rayner, D. M. Villeneuve, and P. B. Corkum, Phys. Rev. Lett. 90, 233003 (2003).

12. D. Pavicic, K. F. Lee, D. M. Rayner, P. B. Corkum, and D. M. Villeneuve, Phys. Rev. Lett. 98, 243001 (2007).

13. K. F. Lee, F. Légaré, D. M. Villeneuve, and P. B. Corkum, J. Phys. B 39, 4081 (2006).

14. P. H. Bucksbaum, A. Zavriyev, H. G. Muller, and D. W. Schumacher, Phys. Rev. Lett. 64, 1883 (1990).

15. J. Ullrich, R. Moshammer, A. Dorn, R. Dörner, L. Ph. H. Schmidt, and H. Schmidt-Böcking, Rep. Prog. Phys. 66, 1463 (2003).

16. X. M. Tong, Z. X. Zhao, and C. D. Lin, Phys. Rev. A 66, 033402 (2002).

17. F. Légaré, K. F. Lee, I. V. Litvinyuk, P. W. Dooley, A. D. Bandrauk, D. M. Villeneuve, and P. B. Corkum, Phys. Rev. A 72, 052717 (2005).

18. J. Itatani, J. Lévesque, D. Zeidler, H. Niikura, H. Pépin, J. C. Kieffer, P. B. Corkum, and D. M. Villeneuve, Nature 432, 867 (2004).

19. P. W. Dooley, I. V. Litvinyuk, K. F. Lee, D. M. Rayner, M. Spanner, D. M. Villeneuve, and P. B. Corkum, Phys. Rev. A 68, 023406 (2003).

20. J. Itatani, D. Zeidler, J. Lévesque, M. Spanner, D. M. Spanner, D. M. Villeneuve, and P. B. Corkum, Phys. Rev. Lett. 94, 123902 (2005).

21. C. Z. Bisgaard (private commun.).

22. K. J. Miller, J. Am. Chem. Soc. 112, 8533 (1990).

23. D. M. Rayner, A. Naumov, and P. B. Corkum, Opt. Express 13, 3208 (2005).

24. P. B. Corkum and F. Krausz, Nature 3, 381 (2007).

25. M. Uiberacker, Th. Uphues, M. Schultze, A. J. Verhoef, V. Yakovlev, M. F. Kling, J. Rauschenberger, N. M. Kabachnik, H. Schröder, M. Lezius, K. L. Kompa, H.-G. Muller, M. J. J. Vrakking, S. Hendel, U. Kleineberg, U. Heinzmann, M. Drescher, and F. Krausz, Nature 446, 627 (2007). 